\title{
Implementation of Backpropagation Artificial Neural Networks to Predict Palm Oil Price Fresh Fruit Bunches
}

\author{
Edi Ismanto $^{1}$, Noverta Effendi ${ }^{1}$, Eka Pandu Cynthia ${ }^{2 *}$ \\ ${ }^{1}$ Muhammadiyah University of Riau \\ ${ }^{2}$ State Islamic University of Sultan Syarif Kasim Riau \\ 1edi.ismanto@umri.ac.id, ${ }^{1}$ nover@umri.ac.id, ${ }^{2}$ eka.pandu.cynthia@uin- \\ suska.ac.id
}

\begin{abstract}
Riau Province is one of the regions known for its plantation products, especially in the oil palm sector, so that Riau Province and regional districts focus on oil palm plants as the main commodity of plantations in Riau. Based on data from the Central Bureau of Statistics (BPS) of Riau Province, the annual production of oil palm plantations, especially smallholder plantations in Riau province has always increased. So is the demand for world CPO. But sometimes the selling price of oil palm fresh fruit bunches (FFB) for smallholder plantations always changes due to many influential factors. With the Artificial Neural Network approach, the Backpropagation algorithm we conduct training and testing of the time series variables that affect the data, namely data on the area of oil palm plantations in Riau Province; Total palm oil production in Riau Province; Palm Oil Productivity in Riau Province; Palm Oil Exports in Riau Province and Average World CPO Prices. Then price predictions will be made in the future. Based on the results of the training and testing, the best Artificial Neural Network (ANN) architecture model was obtained with 9 input layers, 5 hidden layers and 1 output layer. The output of RMSE 0000699 error value and accuracy percentage is $99.97 \%$ so that it can make price predictions according to the given target value.
\end{abstract}

Keywords: Artificial Neural Network, Backpropagation, Palm Oil, Prediction.

\section{Introduction}

Riau Province is one of the regions known for its plantation products, especially in the oil palm sector, so that Riau Province and regional districts focus on oil palm plants as the main commodity of plantations in Riau, based on the first consideration that the land and climate conditions in Riau Province are suitable for oil palm plantations, the second in terms of marketing, Riau Province has ports as marketing transportation access and is adjacent to Malaysia and Singapore as marketing targets. Based on data from BPS Production of plantation products, especially smallholder plantations in Riau province every year has always been increasing, so has world CPO demand, But sometimes the selling price of oil palm FFB for smallholder plantations is always changing, so this needs to be analyzed in the coming year[1]-[3]. Artificial Neural Network[4]-[8] backpropagation is an algorithm for the development of Artificial Neural Networks using backpropagation[9]-[11], we can use multiple screens to train the network to get a balance in pattern recognition. From the description above, this study will try to study and analyze data testing to predict the price of oil palm FFB in the coming year.

\section{Rudimentary}

\subsection{Artificial Neural Network}

Artificial Intelligence is a study of how to make computers do things that can now be done better by humans by using forms of symbols rather than numbers organized and 
analyzed so that they can be understood and applied in decision making and problem solving[1], [12]

\subsection{Backpropogation}

Backpropagation is one part of the Neural Network using the supervised learning model that can minimize errors in the output generated by the network because it uses a multilayer concept[13].

\section{Research Methodology}

In the case of this study, the method used is a descriptive approach or survey that is collecting as much data as possible about the factors related to variable data that affect the price of oil palm FFB, then analyzing these factors and items to find their role in predictions affect the price of Oil Palm FFB in Riau Province.

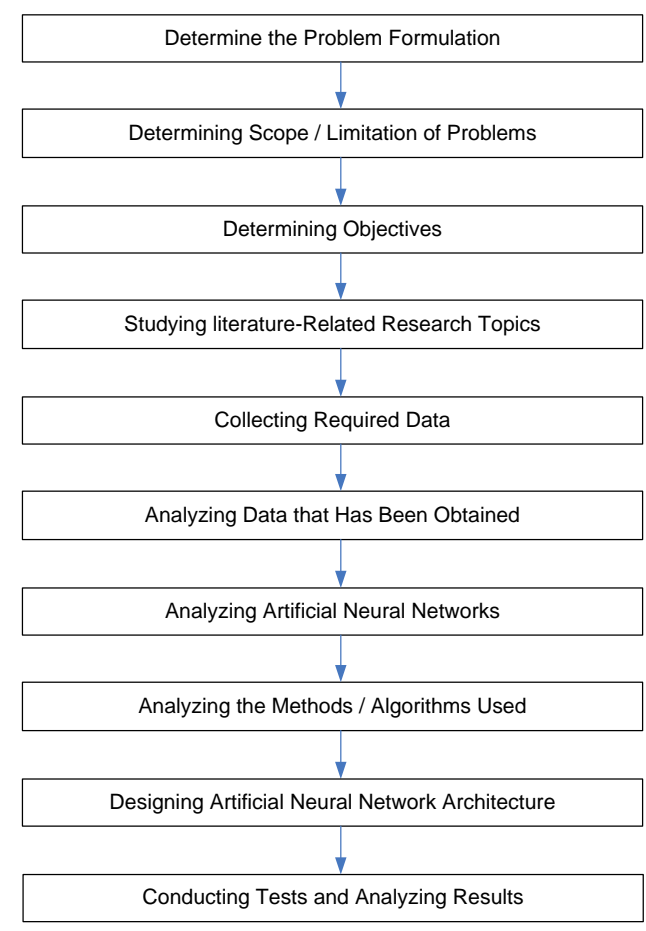

Figure 1. Research Methodology

\section{Result and Discussion}

\subsection{Analysis}

In analyzing data from the field that affects the price of oil palm FFB in Riau province includes five things: Area of Oil Palm Plantation, Palm Oil Production, Riau Palm Oil Productivity, Palm Oil Export, and World CPO Prices. Based on data obtained from the Central Statistics Agency and the Agriculture and Plantation Service of Riau Province from 2008 to 2017 and can be seen in Table 1 as follows:

Table 1. Factors That Affect the Price of FFB

\begin{tabular}{|c|c|c|c|c|c|c|c|c|c|}
\hline \multirow{2}{*}{ Year } & \multicolumn{3}{|c|}{$\begin{array}{c}\text { Area of Riau Palm Oil Plantation } \\
\text { (Unit : Ha) }\end{array}$} & \multicolumn{3}{|c|}{$\begin{array}{c}\text { Riau Palm Oil Production Amount } \\
\text { (Unit : Ton) }\end{array}$} & \multicolumn{3}{|c|}{$\begin{array}{c}\text { Riau Palm Oil Productivity } \\
\text { (Unit : } \mathrm{Kg} / \mathrm{Ha})\end{array}$} \\
\hline & $\begin{array}{l}\text { People's } \\
\text { Plantation }\end{array}$ & $\begin{array}{c}\text { State } \\
\text { Plantation }\end{array}$ & $\begin{array}{c}\text { Private } \\
\text { Plantation }\end{array}$ & $\begin{array}{l}\text { People's } \\
\text { Plantation }\end{array}$ & $\begin{array}{c}\text { State } \\
\text { Plantation }\end{array}$ & $\begin{array}{c}\text { Private } \\
\text { Plantation }\end{array}$ & $\begin{array}{l}\text { People's } \\
\text { Plantation }\end{array}$ & $\begin{array}{c}\text { State } \\
\text { Plantation }\end{array}$ & $\begin{array}{l}\text { Private } \\
\text { Plantation }\end{array}$ \\
\hline$F 1$ & $F 2$ & $F 3$ & F4 & $F 5$ & $F 6$ & F7 & $F 8$ & F9 & $F 10$ \\
\hline$X 1$ & 845,232 & 74,721 & 562,402 & $2,368,076$ & 239,277 & $2,205,532$ & $3,775.41$ & $3,898.92$ & $4,362.82$ \\
\hline$X 2$ & 889,916 & 75,395 & 646,846 & $2,658,044$ & 274,637 & $2,378,687$ & $3,805.33$ & $4,339.34$ & $4,344.00$ \\
\hline$X 3$ & $1,055,543$ & 75,841 & 648,670 & $2,894,459$ & 215,056 & $2,386,453$ & $3,852.49$ & $3,790.00$ & $4,352.34$ \\
\hline
\end{tabular}




\begin{tabular}{|c|c|c|c|c|c|c|c|c|c|}
\hline \multirow{2}{*}{ Year } & \multicolumn{3}{|c|}{$\begin{array}{c}\text { Area of Riau Palm Oil Plantation } \\
\text { (Unit : Ha) }\end{array}$} & \multicolumn{3}{|c|}{$\begin{array}{c}\text { Riau Palm Oil Production Amount } \\
\text { (Unit : Ton) }\end{array}$} & \multicolumn{3}{|c|}{$\begin{array}{c}\text { Riau Palm Oil Productivity } \\
\text { (Unit : } \mathrm{Kg} / \mathrm{Ha})\end{array}$} \\
\hline & $\begin{array}{c}\text { People's } \\
\text { Plantation }\end{array}$ & $\begin{array}{c}\text { State } \\
\text { Plantation }\end{array}$ & $\begin{array}{c}\text { Private } \\
\text { Plantation }\end{array}$ & $\begin{array}{c}\text { People's } \\
\text { Plantation }\end{array}$ & $\begin{array}{c}\text { State } \\
\text { Plantation }\end{array}$ & $\begin{array}{c}\text { Private } \\
\text { Plantation }\end{array}$ & $\begin{array}{c}\text { People's } \\
\text { Plantation }\end{array}$ & $\begin{array}{c}\text { State } \\
\text { Plantation }\end{array}$ & $\begin{array}{c}\text { Private } \\
\text { Plantation }\end{array}$ \\
\hline X4 & $1,205,498$ & 78,979 & 634,551 & $3,174,176$ & 244,393 & $2,330,298$ & $3,595.30$ & $3,826.83$ & $4,134.52$ \\
\hline$X 5$ & $1,297,294$ & 77,740 & 764,154 & $3,485,172$ & 225,124 & $2,674,241$ & $3,644.78$ & $3,775.54$ & $4,052.60$ \\
\hline X6 & $1,348,076$ & 83,670 & 761,975 & $3,692,195$ & 249,321 & $2,705,481$ & $3,413.61$ & $4,014.64$ & $4,024.34$ \\
\hline$X 7$ & $1,357,819$ & 85,586 & 847,331 & $3,706,891$ & 264,791 & $3,021,559$ & $3,373.00$ & $3,943.00$ & $4,048.00$ \\
\hline$X 8$ & $1,354,503$ & 91,854 & 954,519 & $3,611,853$ & 312,012 & $4,135,981$ & $3,228.00$ & $3,878.00$ & $4,974.00$ \\
\hline$X 9$ & $1,360,855$ & 92,130 & 977,523 & $3,651,687$ & 322,970 & $4,531,989$ & $3,235.00$ & $3,890.00$ & $4,996.00$ \\
\hline$X 10$ & $1,386,575$ & 92,714 & $1,013,887$ & $3,677,989$ & 328,159 & $4,715,000$ & $3,251.00$ & $3,928.00$ & $5,105.00$ \\
\hline
\end{tabular}

\begin{tabular}{|c|c|c|c|c|}
\hline \multicolumn{4}{|c|}{$\begin{array}{c}\text { Export of Riau Palm Oil } \\
\text { (Unit : Ton) }\end{array}$} & \multirow{2}{*}{$\begin{array}{c}\text { World CPO Prices } \\
\text { (Unit : \$ / mt) } \\
\\
\text { The Pink Sheet, World Bank }\end{array}$} \\
\hline $\begin{array}{c}\text { CPO } \\
\text { HC : } 151110000\end{array}$ & $\begin{array}{c}\text { OPO } \\
\text { HC : } 151190000 \\
\end{array}$ & $\begin{array}{c}\text { COPK } \\
\text { HC : } 151321000\end{array}$ & $\begin{array}{c}\text { OCOPK } \\
\text { HC : } 151329000 \\
\end{array}$ & \\
\hline F11 & F12 & $F 13$ & F14 & F15 \\
\hline $3,786,818$ & $3,196,042$ & 526,585 & 50,818 & 949 \\
\hline $3,914,362$ & $4,068,195$ & 660,208 & 69,487 & 683 \\
\hline $3,829,333$ & $3,486,760$ & 541,829 & 92,924 & 901 \\
\hline $3,338,428$ & $4,180,607$ & 503,057 & 147,687 & 1,125 \\
\hline $2,783,650$ & $5,346,979$ & 198,606 & 359,393 & 999 \\
\hline $2,566,013$ & $5,525,954$ & 74,433 & 532,193 & 857 \\
\hline $2,115,609$ & $6,595,051$ & 94,327 & 562,397 & 821 \\
\hline $3,249,072$ & $7,512,725$ & 260,789 & 607,532 & 623 \\
\hline $2,821,102$ & $7,014,056$ & 129,062 & 563,959 & 700 \\
\hline $3,035,087$ & $7,263,390$ & 194,925 & 585,745 & 715 \\
\hline
\end{tabular}

\subsection{Data Normalization}

In this study using the binary sigmoid activation function, then the values we will use on artificial neural networks will have to be worth 0 to 1 . The normalization data formula is as follows (Sya'diyah, 2011):

Value $X_{\text {new }}=\frac{\text { Value } X_{\text {old }}-\text { Value } X_{\text {Minimum }}}{\text { Value } X_{\text {Maximum }}-\text { Value } X_{\text {Minimum }}}$

(1)

The results of the data normalization process can be seen in the following table.

Table 2. Normalization Data

\begin{tabular}{|r|r|r|r|r|r|r|r|}
\hline F1 & \multicolumn{1}{|c|}{ F2 } & \multicolumn{1}{c|}{ F3 } & \multicolumn{1}{c|}{ F4 } & \multicolumn{1}{|c|}{ F5 } & \multicolumn{1}{c|}{ F6 } & \multicolumn{1}{|c|}{ F7 } & \multicolumn{1}{c|}{ F8 } \\
\hline X1 & 0 & 0 & 0 & 0 & 0,214150 & 0 & 0,876571 \\
\hline $\mathbf{X 2}$ & 0,082543 & 0,037459 & 0,187036 & 0,216586 & 0,526785 & 0,069001 & 0,924482 \\
\hline $\mathbf{X 3}$ & 0,388499 & 0,062246 & 0,191076 & 0,393171 & 0 & 0,072095 & 1 \\
\hline $\mathbf{X 4}$ & 0,665504 & 0,236648 & 0,159804 & 0,602100 & 0,259383 & 0,049718 & 0,588160 \\
\hline $\mathbf{X 5}$ & 0,835075 & 0,167787 & 0,446863 & 0,834392 & 0,089016 & 0,186776 & 0,667393 \\
\hline $\mathbf{X 6}$ & 0,928882 & 0,497360 & 0,442037 & 0,989023 & 0,302954 & 0,199225 & 0,297219 \\
\hline $\mathbf{X 7}$ & 0,946880 & 0,603846 & 0,631093 & 1 & 0,439732 & 0,325179 & 0,232189 \\
\hline $\mathbf{X 8}$ & 0,940755 & 0,952204 & 0,868505 & 0,929013 & 0,857236 & 0,769266 & 0 \\
\hline $\mathbf{X 9}$ & 0,952489 & 0,967543 & 0,919457 & 0,958767 & 0,954121 & 0,927072 & 0,011209 \\
\hline $\mathbf{X 1 0}$ & 1 & 1 & 1 & 0,978412 & 1 & 1 & 0,036830 \\
\hline
\end{tabular}

\begin{tabular}{|r|c|r|r|r|r|r|}
\hline \multicolumn{1}{|c|}{ F9 } & F10 & \multicolumn{1}{c|}{ F11 } & \multicolumn{1}{|c|}{ F12 } & \multicolumn{1}{|c|}{ F13 } & \multicolumn{1}{|c|}{ F14 } & \multicolumn{1}{|c|}{ F15 } \\
\hline 0,218836 & 0,313216 & 0,929093 & 0 & 0,771887 & 0 & 0,649402 \\
\hline 1 & 0,295801 & 1 & 0,202042 & 1 & 0,033534 & 0,119522 \\
\hline 0,025647 & 0,303518 & 0,952729 & 0,067348 & 0,797910 & 0,075633 & 0,553785 \\
\hline 0,090972 & 0,101956 & 0,679815 & 0,228084 & 0,731721 & 0,174001 & 1 \\
\hline 0 & 0,026151 & 0,371391 & 0,498285 & 0,211981 & 0,554279 & 0,749004 \\
\hline 0,424087 & 0 & 0,250398 & 0,539746 & 0 & 0,864672 & 0,466135 \\
\hline 0,297020 & 0,021894 & 0 & 0,787412 & 0,033962 & 0,918926 & 0,394422 \\
\hline
\end{tabular}




\begin{tabular}{|r|r|r|r|r|r|r|}
\hline 0,181731 & 0,878778 & 0,630138 & 1 & 0,318136 & 1 & 0 \\
\hline 0,203015 & 0,899136 & 0,392212 & 0,884479 & 0,093259 & 0,921732 & 0,153386 \\
\hline 0,270415 & 1 & 0,511175 & 0,942239 & 0,205697 & 0,960865 & 0,183267 \\
\hline
\end{tabular}

\subsection{Artificial Neural Network Design}

In this study an artificial neural network architecture will be made using the backpropagation algorithm to predict the price of oil palm FFB in Riau Province which can be seen in the following figure:

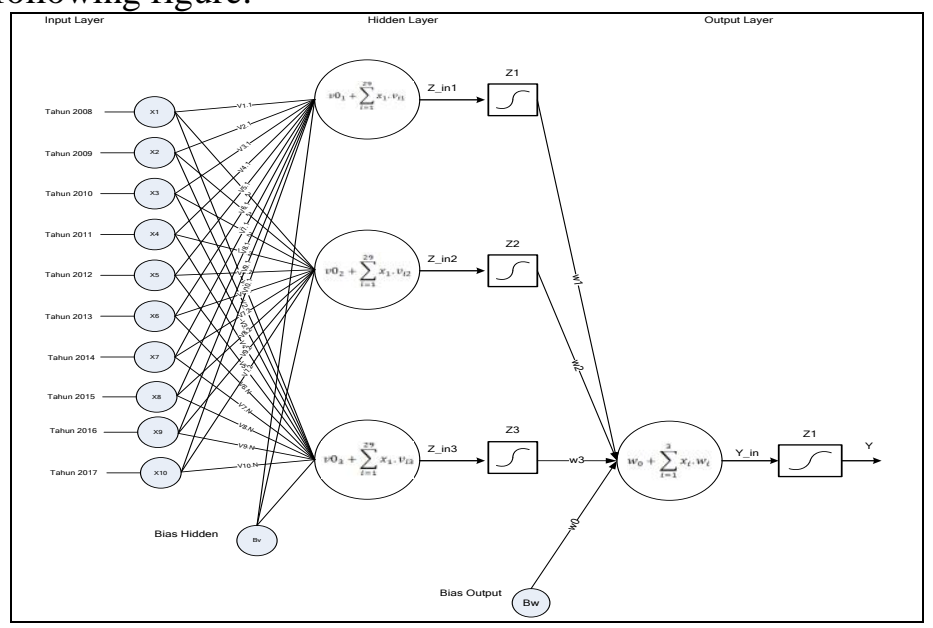

Figure 2. Artificial Neural Network Architecture

\subsection{Training and Testing}

In this study an experiment of several artificial neural network architectures with different parameter settings was conducted. This is done to get the best neural network architecture and parameters.

Table 3. Experiment of Several ANN Architectures

\begin{tabular}{|c|c|c|c|c|c|c|c|c|c|}
\hline \multirow{2}{*}{$\begin{array}{l}\text { Architectural } \\
\text { Pattern }\end{array}$} & \multicolumn{3}{|c|}{ Parameter } & \multicolumn{6}{|c|}{ Training Result } \\
\hline & $\begin{array}{c}\text { Learning } \\
\text { Rate }\end{array}$ & Goal & $\begin{array}{l}\text { Training } \\
\text { Function }\end{array}$ & Epoch & Time & MSE & Gradient & Regression & Accuracy \\
\hline \multirow{5}{*}{ Pola 9 - 3 - 1} & 0,01 & 0,01 & $\begin{array}{c}\text { Logsig } \\
\text { Logsig } \\
\text { Traingdx }\end{array}$ & 144 & 0:00:01 & 0,0096506 & 0,0086506 & 0,96475 & $96,475 \%$ \\
\hline & & & & & & & & & \\
\hline & 0,001 & 0,001 & $\begin{array}{c}\text { Logsig } \\
\text { Logsig } \\
\text { Traingdx }\end{array}$ & 238 & 0:00:00 & 0,0009922 & 0,00064615 & 0,99649 & $99,649 \%$ \\
\hline & & & & & & & & & \\
\hline & 0,0001 & 0,0001 & $\begin{array}{c}\text { Logsig } \\
\text { Logsig } \\
\text { Traingdx }\end{array}$ & 1.083 & 0:00:01 & 0,0095941 & 0,00027638 & 0,99899 & $99,899 \%$ \\
\hline & & & & & & & & & \\
\hline \multirow{5}{*}{ Pola 9 - 5 - 1} & 0,01 & 0,01 & $\begin{array}{c}\text { Logsig } \\
\text { Logsig } \\
\text { Traingdx }\end{array}$ & 158 & 0:00:02 & 0,0094527 & 0,23939 & 0,96585 & $96,585 \%$ \\
\hline & & & & & & & & & \\
\hline & 0,001 & 0,001 & $\begin{array}{c}\text { Logsig } \\
\text { Logsig } \\
\text { Traingdx }\end{array}$ & 364 & 0:00:00 & 0,00099676 & 0,00025621 & 0,99635 & $99,635 \%$ \\
\hline & & & & & & & & & \\
\hline & 0,0001 & 0,0001 & $\begin{array}{c}\text { Logsig } \\
\text { Logsig } \\
\text { Traingdx }\end{array}$ & 1.524 & 0:00:02 & 0,0000699 & 0,00099905 & 0,99970 & $99,970 \%$ \\
\hline
\end{tabular}

From the table above it can be seen that the best artificial neural network architecture is with patterns $9-5-1$ using the learning rate parameter 0,0001 , goal 0,0001 , training 
function Logsig - Logsig - Traingdx. The following are the results of the training and testing using the pattern architecture

Table 4. Result for 9-5-1 ANN Architecture

\begin{tabular}{|l|l|}
\hline \multicolumn{1}{|c|}{ Parameter } & Results \\
1. Learning Rate $/$ Goal $=0,0001 / 0,0001$ & 1. Meet Goal on Epochs ke $=1.524$ \\
2. Maximum Epochs $=100.000$ & 2. Time Duration $=0: 00: 02$ \\
3. Function = Logsig - Logsig - Traingdx & 3. Performance $=0,000999$ \\
& 4. Gradient $=0,000699$ \\
& 5. Percentation of Acuration $=99,97 \%$ \\
\hline
\end{tabular}

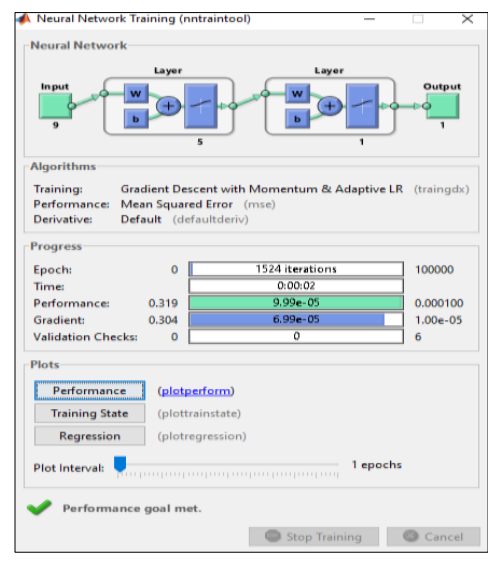

a. Neural Network Training 9-5-1 (c)

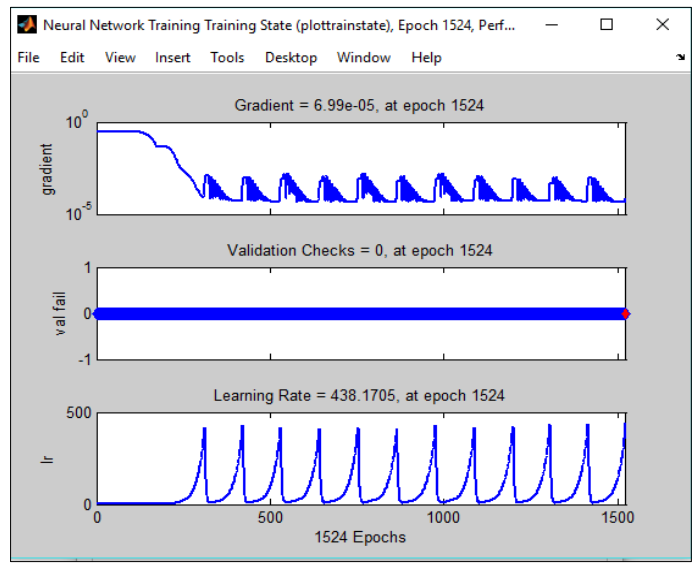

c. Neural Network Training State 9-5-1 (c)

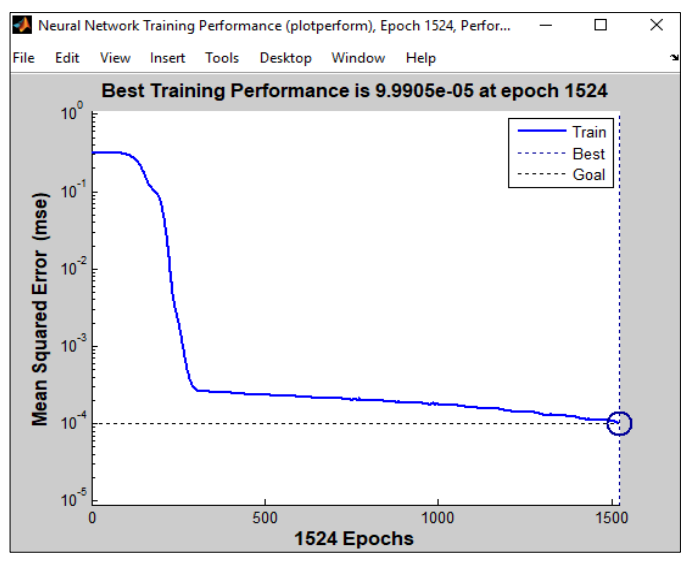

b. Neural Network Training Performance 9-5-1 (c)

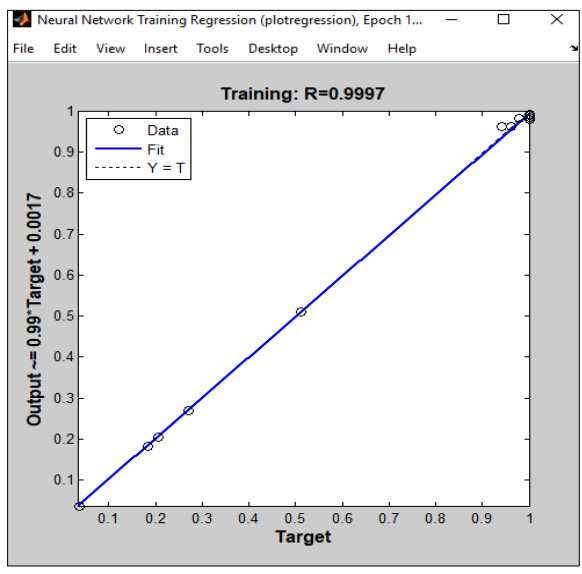

d. Neural Network Training Regression 9-5-1 (c)

Figure 3. Neural Network Training Results

Table 5. Comparison for Output and Target Value

\begin{tabular}{|l|l|l|l|l|l|l|l|l|l|l|l|l|l|l|l|}
\hline $\mathbf{Y}$ & 0,9800 & 0,9892 & 0,9844 & 0,9826 & 0,9908 & 0,9907 & 0,0359 & 0,2704 & 0,9921 & 0,5111 & 0,9617 & 0,2055 & 0,9634 & 0,1833 \\
\hline $\mathbf{T}$ & 1,0000 & 1,0000 & 1,0000 & 0,9784 & 1,0000 & 1,0000 & 0,0368 & 0,2704 & $1 ., 0000$ & 0,5112 & 0,9422 & 0,2057 & 0,9609 & 0,1833 \\
\hline
\end{tabular}

\subsection{Prediction Results and Data Denormalization}

The following are the results of the prediction of the price of oil palm FFB in Riau Province using the data denormalization formula.

$$
x_{i}=y_{n}\left(x_{\max }-x_{\min }\right)+x_{\min }
$$


Table 6. Prediction Result for Area of Riau Palm Oil Plantation

\begin{tabular}{|c|c|c|c|c|}
\hline & & Area of & u Palm Oil Planta & n (Ha) \\
\hline & Year & People's Plantation & State Plantation & Private Plantation \\
\hline & $F_{1}$ & $F_{2}$ & $F_{3}$ & $F_{4}$ \\
\hline & $X_{1}$ & 845232 & 74721 & 562402 \\
\hline & $X_{2}$ & 889.916 & 75.395 & 646.846 \\
\hline Prediction & $X_{3}$ & 1.055 .543 & 75.841 & 648.670 \\
\hline & $X_{4}$ & 1.205 .498 & 78.979 & 634.551 \\
\hline & $X_{5}$ & 1.297 .294 & 77.740 & 764.154 \\
\hline & $X_{6}$ & 1.348 .076 & 83.670 & 761.975 \\
\hline & $X_{7}$ & 1.357 .819 & 85.586 & 847.331 \\
\hline & $X_{8}$ & 1.354 .503 & 91.854 & 954.519 \\
\hline & $X_{9}$ & 1.360 .855 & 92.130 & 977.523 \\
\hline & $X_{10}$ & 1.386 .575 & 92.714 & 1.013 .887 \\
\hline Yn: & & 0,9800 & 0,9892 & 0,9844 \\
\hline Min : & 2008 & 845.232 & 74.721 & 562.402 \\
\hline Max: & 2017 & 1.386 .575 & 92.714 & 1.013 .887 \\
\hline $\begin{array}{c}\text { Prediction } \\
\text { Result }\end{array}$ & & $1.375 .748,1400$ & $92.519,6756$ & $1.006 .843,8340$ \\
\hline $\begin{array}{c}\text { Conclusion } \\
\text { Comparison } \\
\text { with last } \\
\text { year }(2017)\end{array}$ & 2018 & DECREASE & DECREASE & DECREASE \\
\hline
\end{tabular}

Table 7. Prediction Result for Riau Palm Oil Production Amount

\begin{tabular}{|c|c|c|c|c|}
\hline \multirow{13}{*}{ Prediction } & \multirow{2}{*}{ Year } & \multicolumn{3}{|c|}{ Riau Palm Oil Production Amount (Ton) } \\
\hline & & People's Plantation & State Plantation & Private Plantation \\
\hline & $F_{1}$ & $F_{5}$ & $F_{6}$ & $F_{7}$ \\
\hline & $\mathrm{X}_{1}$ & 2.368 .076 & 239.277 & 2.205 .532 \\
\hline & $X_{2}$ & 2.658 .044 & 274.637 & 2.378 .687 \\
\hline & $X_{3}$ & 2.894 .459 & 215.056 & 2.386 .453 \\
\hline & $X_{4}$ & 3.174 .176 & 244.393 & 2.330 .298 \\
\hline & $X_{5}$ & 3.485 .172 & 225.124 & 2.674 .241 \\
\hline & $X_{6}$ & 3.692 .195 & 249.321 & 2.705 .481 \\
\hline & $X_{7}$ & 3.706 .891 & 264.791 & 3.021 .559 \\
\hline & $X_{8}$ & 3.611 .853 & 312.012 & 4.135 .981 \\
\hline & $X_{9}$ & 3.651 .687 & 322.970 & 4.531 .989 \\
\hline & $X_{10}$ & 3.677 .989 & 328.159 & 4.715 .000 \\
\hline Yn: & & 0,9826 & 0,9908 & 0,9907 \\
\hline Min : & 2008 & 2.368 .076 & 239.277 & 2.205 .532 \\
\hline Max: & 2017 & 3.677 .989 & 328.159 & 4.715 .000 \\
\hline $\begin{array}{c}\text { Prediction } \\
\text { Result }\end{array}$ & & $3.655 .196,5138$ & $327.341,2856$ & $4.691 .661,9476$ \\
\hline $\begin{array}{c}\text { Conclusion } \\
\text { Comparison } \\
\text { with last } \\
\text { year (2017) }\end{array}$ & 2018 & DECREASE & DECREASE & DECREASE \\
\hline
\end{tabular}


Table 8. Prediction Result for Riau Palm Oil Productivity

\begin{tabular}{|c|c|c|c|c|}
\hline \multirow{13}{*}{ Prediction } & \multirow{2}{*}{ Year } & \multicolumn{3}{|c|}{ Riau Palm Oil Productivity (Kg/Ha) } \\
\hline & & People's Plantation & State Plantation & Private Plantation \\
\hline & $F_{1}$ & $F_{8}$ & $F_{9}$ & $F_{10}$ \\
\hline & $X_{1}$ & $3.775,41$ & $3.898,92$ & $4.362,82$ \\
\hline & $X_{2}$ & $3.805,33$ & $4.339,34$ & $4.344,00$ \\
\hline & $X_{3}$ & $3.852,49$ & $3.790,00$ & $4.352,34$ \\
\hline & $X_{4}$ & $3.595,30$ & $3.826,83$ & $4.134,52$ \\
\hline & $X_{5}$ & $3.644,78$ & $3.775,54$ & $4.052,60$ \\
\hline & $X_{6}$ & $3.413,61$ & $4.014,64$ & $4.024,34$ \\
\hline & $X_{7}$ & $3.373,00$ & $3.943,00$ & $4.048,00$ \\
\hline & $X_{8}$ & $3.228,00$ & $3.878,00$ & $4.974,00$ \\
\hline & $X_{9}$ & $3.235,00$ & $3.890,00$ & $4.996,00$ \\
\hline & $X_{10}$ & $3.251,00$ & $3.928,00$ & $5.105,00$ \\
\hline Yn: & & 0,0359 & 0,2704 & 0,9921 \\
\hline Min : & 2008 & $3.775,4100$ & $3.898,9200$ & $4.362,8200$ \\
\hline Max: & 2017 & $3.251,0000$ & $3.928,0000$ & $5.105,0000$ \\
\hline $\begin{array}{c}\text { Prediction } \\
\text { Result }\end{array}$ & & $3.756,5837$ & $3.906,7832$ & $5.099,1368$ \\
\hline $\begin{array}{c}\text { Conclusion } \\
\text { Comparison } \\
\text { with last year } \\
(2017)\end{array}$ & 2018 & INCREASE & DECREASE & DECREASE \\
\hline
\end{tabular}

Table 9. Prediction Result for Export of Riau Palm Oil

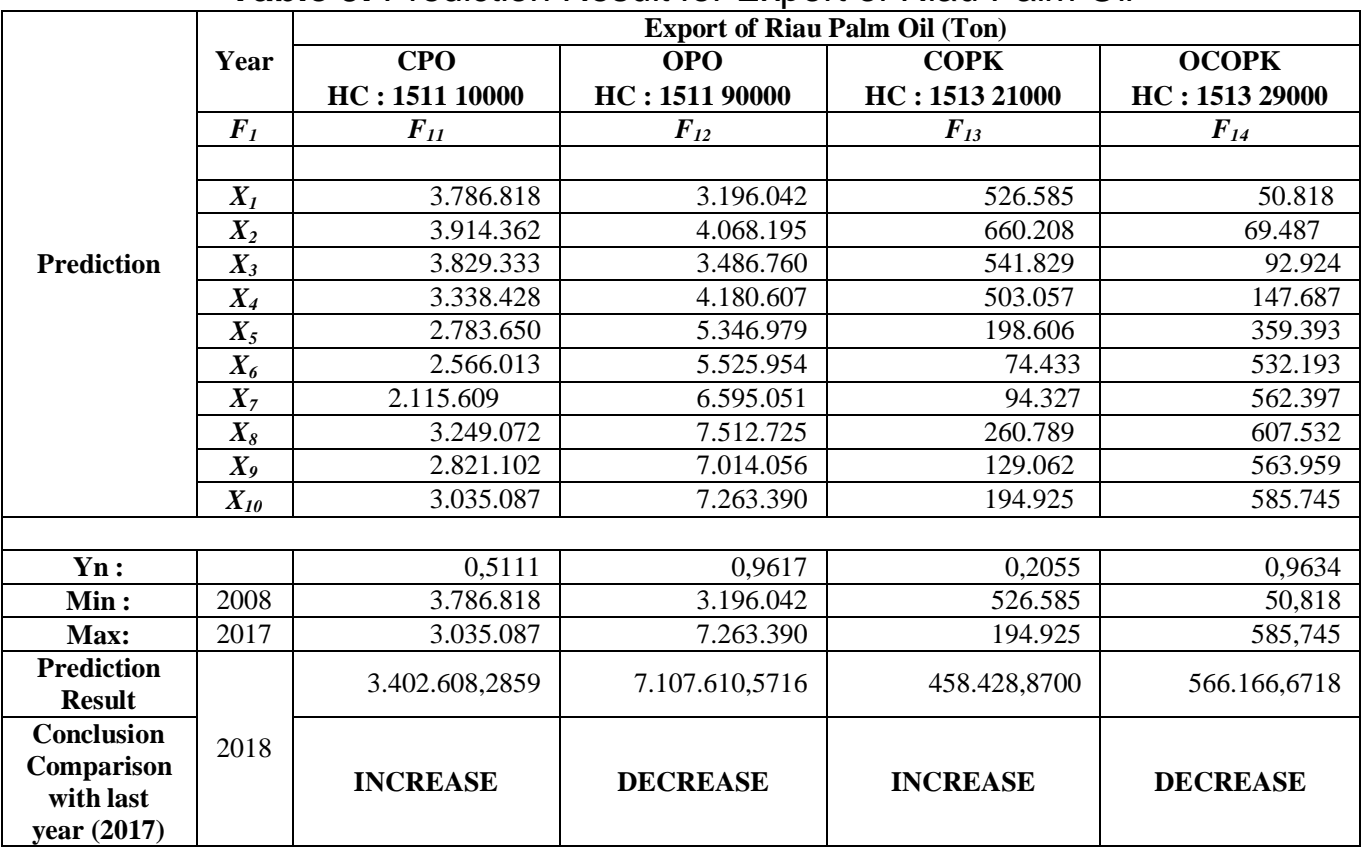


Table 10. Prediction Result for World CPO Prices

\begin{tabular}{|c|c|c|}
\hline \multirow{13}{*}{ Prediction } & \multirow[t]{2}{*}{ Year } & $\begin{array}{c}\text { World CPO Prices } \\
\text { (Unit }: \$ / \mathrm{mt})\end{array}$ \\
\hline & & The Pink Sheet, World Bank \\
\hline & $F_{1}$ & $F_{15}$ \\
\hline & $X_{1}$ & 949 \\
\hline & $X_{2}$ & 683 \\
\hline & $X_{3}$ & 901 \\
\hline & $X_{4}$ & 1.125 \\
\hline & $X_{5}$ & 999 \\
\hline & $X_{6}$ & 857 \\
\hline & $X_{7}$ & 821 \\
\hline & $X_{8}$ & 623 \\
\hline & $X_{9}$ & 700 \\
\hline & $X_{10}$ & 715 \\
\hline Yn : & & 0,1833 \\
\hline Min : & 2008 & 949 \\
\hline Max: & 2017 & 715 \\
\hline Prediction Result & & 906,1078 \\
\hline $\begin{array}{c}\text { Conclusion } \\
\text { Comparison with last year (2017) }\end{array}$ & 2018 & INCREASE \\
\hline
\end{tabular}

\section{Conclusion}

After going through the testing phase for artificial neural network architecture using backpropagation algorithm, a conclusion can be drawn:

a. Artificial Neural Networks (ANN) Backpropagation can produce the best architectural patterns in data pattern recognition so that the Artificial Neural Network method can be used to predict the price of Oil Palm FFB in Riau Province.

b. Variable learning rate, the amount of learning data and the weight value used can influence the level of truth predictions so that it needs to be analyzed correctly in using data variables.

c. Based on the results of training and artificial neural network testing, the best architectural model chosen and having the smallest RMSE value is the 9-5-1 architecture with an RMSE error value of 0.0000699 , an accuracy percentage of 99.97\%. The conclusion of the training and testing of the data shows that the prediction of the price of oil palm FFB in 2018 will increase. And this architectural model can be used for consideration in predicting the price of oil palm FFB in Riau Province.

\section{References}

[1] A. Ehret, D. Hochstuhl, D. Gianola, and G. Thaller, "Application of neural networks with back-propagation to genome-enabled prediction of complex traits in HolsteinFriesian and German Fleckvieh cattle," Genet. Sel. Evol., vol. 47, no. 1, p. 22, 2015.

[2] D. Huang and $\mathrm{Z}$. Wu, "Forecasting outpatient visits using empirical mode decomposition coupled with backpropagation artificial neural networks optimized by particle swarm optimization," PLoS One, vol. 12, no. 2, pp. 1-17, 2017.

[3] N. M. Nawi, a. Khan, M. Z. Rehman, M. a. Aziz, J. H. Abawajy, and T. Herawan, "Neural network training by hybrid accelerated cuckoo particle swarm optimization algorithm," Lect. Notes Comput. Sci. (including Subser. Lect. Notes Artif. Intell. Lect. Notes Bioinformatics), vol. 8835, pp. 237-244, 2014.

[4] J. Tarigan, R. Diedan, and Y. Suryana, "Plate Recognition Using Backpropagation Neural Network and Genetic Algorithm," Procedia Comput. Sci. Comput. Intell. ICCSCI, vol. 116, no. 2, pp. 365-372, 2017.

[5] A. Wanto, M. Zarlis, Sawaluddin, D. Hartama, J. Tata Hardinata, and H. F. Silaban, 
"Analysis of Artificial Neural Network Backpropagation Using Conjugate Gradient Fletcher Reeves In The Predicting Process," J. Phys. Conf. Ser., vol. 930, no. 1, pp. 1-7, 2017.

[6] Sumijan, A. P. Windarto, A. Muhammad, and Budiharjo, "Implementation of Neural Networks in Predicting the Understanding Level of Students Subject," Int. J. Softw. Eng. Its Appl., vol. 10, no. 10, pp. 189-204, 2016.

[7] I. Sina and W. Nim, "Penggunaan Graf dalam Algoritma Semut untuk Melakukan Optimisasi."

[8] I. T. R. Yanto, M. A. Ismail, and T. Herawan, "A modified Fuzzy k-Partition based on indiscernibility relation for categorical data clustering," Eng. Appl. Artif. Intell., vol. 53, pp. 41-52, 2016.

[9] A. P. Windarto, L. S. Dewi, and D. Hartama, "Implementation of Artificial Intelligence in Predicting the Value of Indonesian Oil and Gas Exports With BP Algorithm," Int. J. Recent Trends Eng. Res., vol. 3, no. 10, pp. 1-12, 2017.

[10] N. M. Nawi, M. Z. Rehman, M. A. Aziz, T. Herawan, and J. H. Abawajy, "Levenberg Marquardt Back Propagation Algorithm," pp. 245-253.

[11] A. Gupta and M. Shreevastava, "Medical Diagnosis using Back propagation Algorithm," Int. J. Emerg. Technol. Adv. Eng., vol. 1, no. 1, pp. 55-58, 2011.

[12] S. Sumijan, A. Windarto, A. Muhammad, and B. Budiharjo, "Implementation of Neural Networks in Predicting the Understanding Level of Students Subject," Int. J. Softw. Eng. Its Appl., vol. 10, no. 10, pp. 189-204, 2016.

[13] A. Wanto, M. Zarlis, Sawaluddin, and D. Hartama, "Analysis of Artificial Neural Network Backpropagation Using Conjugate Gradient Fletcher Reeves in the Predicting Process," J. Phys. Conf. Ser., vol. 930, no. 1, pp. 1-7, 2017.

\section{Authors}

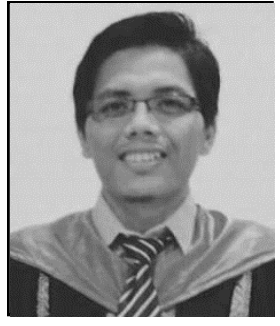

Edi Ismanto, S.T., M.Kom, born in Pasir Pengaraian, Riau. Completed S1 education in the Informatics Engineering Department, UIN Sultan Syarif Kasim Riau (2010). And master's degree in Master of Computer Science at Putra Indonesia University YPTK Padang (2012). Now working as a lecturer in the Department of Informatics, Faculty of Teacher Training and Education, University Muhammadiyah of Riau. With research interests in the field of artificial intelligence.

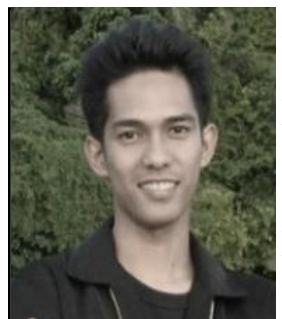

Noverta Effendi, S.T., M.Kom, born in Sungai Kayu Ara. He completed his undergraduate education in the Department of Electrical Engineering, UIN Sultan Syarif Kasim Riau (2008). And master's degree in Master of Computer Science at Putra Indonesia University YPTK Padang (2013). Now working as a lecturer in the Informatics Engineering Department, Faculty of Science and Technology, Sultan Syarif Kasim Islamic State University of Riau. With research interests in computing and computer electronics.

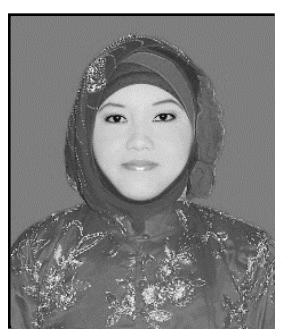

Eka Pandu Cynthia, S.T., M.Kom, born in Pekanbaru, Riau. Completed S1 education in the Informatics Engineering Department, UIN Sultan Syarif Kasim Riau (2011). And master's degree in Master of Computer Science Putra Indonesia University YPTK Padang (2014). Now working as a lecturer in the Informatics Engineering Department, Faculty of Science and Technology, Sultan Syarif Kasim Islamic State University of Riau. With research interests in the field of artificial intelligence. 